CLINICAL STUDY

\title{
Sexual desire in female-to-male transsexual persons: exploration of the role of testosterone administration
}

\author{
Katrien Wierckx ${ }^{1, *}$, Els Elaut ${ }^{2, *}$, Eva Van Caenegem ${ }^{1}$, Fleur Van De Peer ${ }^{1}$, David Dedecker ${ }^{1}$, Ellen Van Houdenhove ${ }^{1}$ \\ and Guy T'Sjoen ${ }^{1}$ \\ ${ }^{1}$ Department of Endocrinology and ${ }^{2}$ Center for Sexology and Gender Problems, Ghent University Hospital, De Pintelaan 185, 9000 Ghent, Belgium \\ (Correspondence should be addressed to K Wierckx; Email: Katrien.Wierckx@ugent.be)
}

*(K Wierckx and E Elaut contributed equally to this work)

\begin{abstract}
Objective: To describe sexual desire in female-to-male transsexual persons post sex reassignment surgery (SRS). The associations between serum androgen levels and sexual desire are examined. Design: Single center cross-sectional study.

Methods: Forty-five female-to-male transsexual persons post SRS completed a standardized questionnaire assessing sexual desire (Sexual Desire Inventory). In addition, participants were asked questions on sexual desire before starting hormone treatment and having SRS. Serum levels of testosterone, LH and sex hormone-binding globulin were measured on fasting morning serum samples.

Results: In retrospect, $73.9 \%$ of the participants reported an increase in sexual desire after hormone treatment and SRS. Solitary sexual desire scores were significantly correlated with frequency of masturbation $(r=0.835 ; P<0.001)$, whereas frequency of sexual intercourse with a partner was not. No direct associations were found between testosterone and solitary or dyadic sexual desire. However, ANOVA showed an independent effect of LH on solitary sexual desire $(P<0.001)$. Post hoc analysis revealed that female-to-male transsexual persons with elevated levels of LH, indicating suboptimal testosterone therapy, reported significantly lower solitary sexual desire levels (than those with low LH levels; $P=0.007)$. Suppressed LH levels were also associated with having a higher need for sexual activities $(P=0.009)$ and a higher frequency of excessive sexual desire $(P=0.007)$.

Conclusion: Most female-to-male transsexual persons report on a marked increase in sexual desire after testosterone treatment and SRS. No direct associations between levels of testosterone and solitary or dyadic sexual desire were found. However, measures of sexual desire were inversely associated with LH levels.
\end{abstract}

European Journal of Endocrinology $165331-337$

\section{Introduction}

It is now widely believed that testosterone plays an important role in motivational aspects of sexual functioning as sexual desire (1). Testosterone therapy seems to enhance the frequency of sexual thoughts and the intensity of sexual feelings, especially in young hypogonadal men $(2,3)$.

During the process of sex reassignment, transsexual persons receive cross-sex hormone replacement that transforms their hormonal milieu. After sex reassignment surgery (SRS), which often includes gonadectomy, cross-sex hormone treatment is continued as in other hypogonadal subjects. In view of a possible impact of testosterone on sexual desire, it has been suggested that the effect of continuous testosterone administration in female-to-male transsexual persons might lead to an increase in sexual desire (4). Also, care givers working in treatment centers for gender dysphoria sometimes encounter complaints of low sexual desire in maleto-female transsexual persons while this is hardly the case in female-to-male transsexual persons. A previous study from our group found that hypoactive sexual desire was reported in one-third of transsexual women. While levels of free testosterone in the group of maleto-female transsexual persons were markedly lower, the intensity and frequency of sexual desire was very similar to control women not using hormonal contraception. These findings argue against a major role of testosterone in the sexual desire in male-to-female transsexuals (5). In a pilot study, Kronawitter et al. (6) treated hypoactive sexual desire in male-to-female transsexual persons with a testosterone patch. A significant improvement in sexual desire (as measured by the Brief Profile of Female Sexual Function) was observed.

Our knowledge of sexual desire in female-to-male transsexual persons is even more limited since data from validated questionnaires on sexual desire in 
female-to-male transsexual persons are nonexistent (4). The available data can only be interpreted as an indication of sexual desire through measurement of the frequency of sexual activity or frequency of masturbation before and after SRS. In their sample of adult female-to-male transsexual persons, De Cuypere et al. (7) generally found an increase in frequency of masturbation after testosterone treatment and SRS. Similarly, Lief \& Hubschmann (8) mentioned an increase in sexual activity following SRS. In their adolescent female-to-male sample, Smith et al. (9) and Cohen-Kettenis \& van Goozen (10) reported no change or an increase in masturbation after testosterone treatment and SRS.

This study aimed at providing a validated measure of sexual desire in this population (11). In addition, we hypothesized a marked association between serum androgen levels and levels of sexual desire in femaleto-male transsexual persons.

\section{Materials and methods}

\section{Study population}

All Dutch speaking female-to-male transsexual persons who received SRS between 1987 and 2009 at our hospital were invited by letter $(n=79)$, in which they were asked to confirm their participation by telephone or electronic mail. Two participants could not be reached due to change of address. Those who had not replied after 1 month were contacted by telephone or were left a voice message as a reminder. Potential participants were contacted a second time if necessary. In this study, a total number of 47 persons agreed to participate, which included a 1 day hospital visit, resulting in a response rate of $64 \%$. Three participants, were informed by others and offered to participate in the study themselves, resulting in a study sample of 50 .

Exclusion criteria included treatments or disorders affecting sex hormone status, body composition, and bone metabolism: untreated hypo- or hyper-thyroidism, Cushing's syndrome, alcohol abuse, mucoviscidosis, malabsorption or eating disorders, cirrhosis, chronic kidney failure, autoimmune rheumatoid arthritis, the current $(<2$ years) or prolonged use of corticosteroids, estrogens, antiandrogens, bisphosphonates, calcitonin and fluorides. The 1st year, after SRS is often called the honeymoon period; a period that does not represent a realistic picture of long-term sexual and psychological status. Participants, who underwent SRS during the last year, were therefore excluded. One participant had to be excluded based on these criteria. Furthermore, four participants were not willing to complete the Sexual Desire Inventory, resulting in a final study population of 45 for this paper.

In our center, female-to-male transsexual persons are treated through a multidisciplinary approach consisting of hormone therapy and SRS for most.
SRS in female-to-male transsexual persons includes mastectomy, hysterectomy, and bilateral ovariectomy. Owing to the extensive experience in phalloplasty at our center $(12,13)$, most female-to-male transsexual persons in this study $(n=38)$ immediately proceeded with a phalloplasty and less frequently, with metaidoioplasty. The majority of participants who initially chose metaidoioplasty $(n=9)$ proceeded toward phalloplasty afterwards $(n=8)$. Two female-to-male transsexual persons underwent only mastectomy, hysterectomy, and bilateral ovariectomy. They had not yet made up their minds about having genital surgery. All participants started hormonal therapy at least 2 years before SRS. On average, participants were 8 years after SRS, with a minimum of 2 and a maximum of 22 years. All participants were on long-term testosterone therapy. While current cross-sex hormone therapy is not standardized, almost all participants were treated by the same endocrinologist. Cross-sex hormone therapy consisted of: i.m. testosterone treatment (parental testosterone esters $250 \mathrm{mg} / 2$ or 3 weeks; $n=32$ ); testosterone undecanoate $1000 \mathrm{mg} / 12$ weeks $(n=7)$; and transdermal testosterone gel (50 mg daily; $n=5$ ). One participant used both oral testosterone undecanoate $40 \mathrm{mg}$ (daily) and transdermal testosterone gel $50 \mathrm{mg}$ (daily).

We chose not to include community-dwelling men, women, or male-to-female transsexual persons as a control group since hormonal status, psychosocial factors and sexual functioning are very specific in this study population.

To contextualize the levels of sexual desire in the present sample, we will use previous data of our research group on sexual desire in community-dwelling men (E Elaut, A Buysse, P De Sutter, G De Cuypere, J Gerris, E Deschepper, G T'Sjoen, Personal Communication) and male-to-female transsexual persons (5) using the same validated questionnaire. The data on sexual desire in communitydwelling men consist of baseline measures collected during a study in 55 heterosexual couples in which the effect of three different forms of hormonal contraception on female sexual desire was assessed (E Elaut, A Buysse, P De Sutter, G De Cuypere, J Gerris, E Deschepper, G T'Sjoen, Personal Communication); data from the male-to-female transsexual persons were collected during a previous study of our group on sexual desire and the association with testosterone (5).

\section{Study procedures}

In this study, female-to-male transsexual persons, who agreed to participate, received all questionnaires by regular mail. Subsequently, they visited the Ghent University Hospital between November 2009 and April 2010 for further evaluation. The visit included a fasting morning blood sample, dermatologic, urological, speech, bone, and body composition evaluations; data that will be reported in other publications (14). 
This study complied with the recommendations of the Declaration of Helsinki and was approved by the Ethics Committee of the Ghent University Hospital. Informed consent was obtained from all participants.

\section{Measures}

Sexual desire Sexual desire was measured using the Dutch version of the Sexual Desire Inventory (11). This self-report questionnaire contains 14 items. Subscales measure the intensity and frequency of the desire to behave sexually with a partner (dyadic sexual desire) or by oneself (solitary sexual desire). For the frequency items, participants chose one out of seven options. For the strength items, participants scored their sexual desire on a nine-point Likert scale ranging from 0 (no desire) to 8 (strong desire). The participants were asked to take the previous month as a reference. Adding items resulted in a score for dyadic and solitary sexual desire. Higher scores indicate a higher level of sexual desire with a maximum score of 62 for the dyadic subscale and 23 for the solitary subscale. The Sexual Desire Inventory has a good reliability and validity (11). Internal consistency in this study population was high (Cronbach's $\alpha=0.89$ ).

Other items concerning sexual desire and sexual functioning in the past month were added: frequency of experiencing sexual desire, frequency of experiencing sexual desire toward their partner, frequency of sexual activities, frequency of masturbation (five-point Likert scale from not at all to daily), current sexual desire compared with after sex reassignment (five-point Likert scale from much higher to much lower), frequency of experiencing excessive sexual desire (five-point Likert scale from almost never to almost always), time one can live without any sexual activities (nine-point Likert scale from always to less than a day), and sexual satisfaction with the current partner (five-point Likert scale from very unsatisfied to very satisfied or not applicable).

\section{Biochemical determinations}

Venous blood samples were obtained between 0800 and $1200 \mathrm{~h}$ after overnight fasting, due to practical reasons regardless of the timing of testosterone administration. All blood samples were stored at $-80{ }^{\circ} \mathrm{C}$ until batch analysis.

Commercial kits for RIA were used to determine the serum concentrations of total testosterone and sex hormone-binding globulin (SHBG; Orion Diagnostica, Espoo, Finland); LH (electrochemiluminescence immunoassay; Modular, Roche Diagnostics. Intra- and inter-assay coefficient of variations were $<10-15 \%$ for all measurements respectively. For all measurements, samples from female-to-male transsexual persons were assayed in a same assay run. Serum-free testosterone was calculated from the total serum hormone concentration, serum SHBG and serum albumin, using a validated equation derived from the mass action law (15). We defined supra- and sub-physiological levels of testosterone and LH as hormone levels exceeding the upper or lower limit of the reference ranges according to values of our local laboratory.

\section{Statistical analysis}

The normal distribution of all variables was tested by the Kolmogorov-Smirnov one-sample test. Normally distributed variables are described in terms of mean and S.D. and skewed variables in terms of median, first and third quartiles. The Kruskall-Wallis test was used to determine differences in the hormone levels according to type of testosterone treatment and to determine differences in sexual desire among female-to-male transsexual persons, male-to-female transsexual persons, and community-dwelling men. ANOVA was used to explore associations between hormonal levels (testosterone, free testosterone, and LH) and measures of sexual desire. Post hoc analyses were performed by LSD tests. The Mann-Whitney $U$ test was used to test differences in sexual desire between participants with testosterone levels above the P75 and below the P25, participants with supra- or subphysiological levels of testosterone and the rest of the group.

All hypothesis tests were two-sided. The level indicating statistical significance was 0.05. Internal consistency within a set of items was assessed through Cronbach's $\alpha$ metric. Statistical analyses were performed by PASW (version 18, 0; SPSS Inc., Chicago, IL, USA).

\section{Results}

\section{Patient characteristics}

Patient characteristics are summarized in Table 1. Twelve participants reported one or more chronic disease(s): autoimmune hypothyroidism $(n=3)$, hypercholesterolemia $(n=2)$, obesity $(n=2)$, hypertension $(n=3)$, liver function problems $(n=2)$, epilepsy, migraine, colitis ulcerosa, psoriasis, Graves' disease, chronic fatigue syndrome, and unspecified disease $(n=1)$.

Table 1 Patient characteristics $(n=45)$.

\begin{tabular}{lcc}
\hline & Mean (s.D.) & Range \\
\hline Age (years) & $37(8.17)$ & $22-54$ \\
Age at time of SRS (years) & $30(7.68)$ & $16-44$ \\
Weight $(\mathrm{kg})$ & $69.17(12.04)$ & $45.0-98.5$ \\
Height $(\mathrm{cm})$ & $165.11(6.73)$ & $147.4-183.9$ \\
Body mass index $\left(\mathrm{kg} / \mathrm{m}^{2}\right)$ & $25.33(3.88)$ & $18.3-34.0$ \\
Active smoking $(\%)$ & 37 & \\
Stopped smoking (\%) & 34.8 & \\
Use of testosterone therapy (\%) & 100 & \\
Duration of testosterone & $9.42(5.82)$ & $3.0-27.0$ \\
$\quad$ therapy (years) & & \\
\hline
\end{tabular}




\section{Sexual desire and sexual functioning}

All but four participants completed the Sexual Desire Inventory. In two questionnaires one item was missing, we inserted there the personal mean of the other items of this subscale. The majority of the participants mentioned an increase in sexual desire after SRS: $38.6 \%(n=17)$ described the current sexual desire as much higher; $34.1 \%(n=15)$ as higher. On the contrary, $25.0 \%(n=11)$ described no change in sexual desire after SRS and one participant mentioned a decrease in sexual desire. One participant was not willing to answer this question.

Most participants masturbated after SRS. Sixty percent said they masturbated weekly or daily. A quarter masturbated once or twice a month. A minority masturbated less than a month $(4.4 \%)$ or not at all $(11.1 \%)$. Frequency of masturbation strongly correlated with solitary $(r=0.803 ; P<0.001)$ and dyadic sexual desire $(r=0.466 ; P=0.001)$. Up to onethird $(32.1 \%)$ of the participants currently in a relationship reported to have sexual intercourse several times a week. Almost half of the participants (48.1\%) reported to have sexual intercourse once or twice a month. Twenty-two percent of the participants were not sexually active with their partner in the past month. Frequency of sexual intercourse was not correlated with either dyadic $(r=0.201 ; P=0.315)$ or solitary sexual desire $(r=0.204 ; P=0.307)$. Sixty-four percent of the participants currently in a relationship expressed their satisfaction with their sexual life, 18\% remained neutral, whereas $18 \%$ expressed to be (very) unsatisfied.

Comparison of female-to-male, male-to-female transsexual persons, and community-dwelling men shows a difference in dyadic sexual desire (Kruskal-Wallis test; $P<0.001)$ but not in solitary sexual desire (Kruskal-Wallis test; $P=0.074$ ). Further analysis shows that female-to-male transsexual persons score significantly higher on solitary and dyadic sexual desire compared with male-to-female transsexual persons but score similarly as community-dwelling men (Fig. 1).

\section{Hormonal data}

As expected, the time between the last testosterone administration and the date of visit was significantly different according to the type of testosterone replacement therapy $(P<0.001)$. Consequently, further ANOVA were corrected for this parameter. We could not detect a statistical difference in the levels of total testosterone, calculated free testosterone, LH, and hematocrit between groups based on the type of testosterone replacement therapy (Table 2).

Testosterone treatment in female-to-male transsexual persons aims at testosterone concentrations in the normal physiological testosterone range for men. At the time of measurement, almost 9\% (8.9\%) had levels

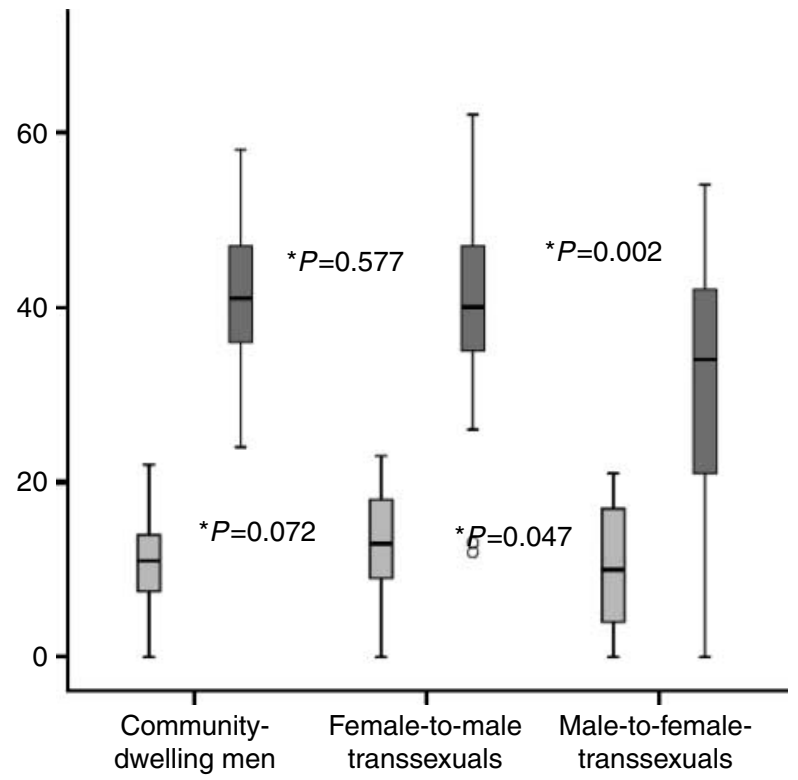

Figure 1 Sexual desire scores. Light grey square, solitary sexual desire; dark grey square, dyadic sexual desire; *Mann-Whitney $U$ test between female-to-male transsexuals and community-dwelling men or male-to-female transsexuals.

below the reference values of our laboratory (testosterone $<321 \mathrm{ng} / \mathrm{dl}$ ), whereas $26.7 \%$ exceeded the upper limit of $1005 \mathrm{ng} / \mathrm{dl}$.

All participants with testosterone values below $321 \mathrm{ng} / \mathrm{dl}$ were treated with classical testosterone esters. No difference was observed in type of testosterone therapy in participants with testosterone above the upper limit.

\section{Association between sexual desire and hormonal levels}

No associations were found between levels of free testosterone (FT) and solitary or dyadic sexual desire by ANOVA after correction for the type of testosterone replacement therapy and time since last testosterone administration (testosterone undecanoate $1000 \mathrm{mg}$ : $P=0.885$; parental testosterone esters $250 \mathrm{mg}$ : $P=0.316$; and transdermal testosterone gel: $P=0.709$ ).

Also, no significant associations were observed between total testosterone (TT) and solitary or dyadic sexual desire (data not shown). We found no differences in measures of solitary or dyadic sexual desire between participants with total or free testosterone levels below P25 and higher than P75 (Mann-Whitney $U$ test: TT, $P=0.30 ; P=0.60$ FT, $P=0.24 ; P=0.40$ ). Also, no differences were observed in measures of sexual desire between participants with supra- or subphysiological testosterone levels in comparison with testosterone levels within the normal range (data not shown).

Supraphysiological LH levels are assumed to reflect inadequate hormone therapy while suppressed levels indicate an excess of sex hormone substitution. Hence, 
Table 2 Serum hormone levels according to the type of testosterone replacement therapy.

\begin{tabular}{|c|c|c|c|c|c|}
\hline & \multicolumn{3}{|c|}{ Testosterone } & \multirow[b]{2}{*}{$\boldsymbol{P}^{\mathrm{a}}$} & \multirow[b]{2}{*}{$\begin{array}{l}\text { Reference } \\
\text { values }^{b}\end{array}$} \\
\hline & $\begin{array}{l}\text { Undecanoate } \\
(1000 \mathrm{mg}, n=7)\end{array}$ & $\begin{array}{l}\text { Parental esters } \\
(250 \mathrm{mg}, n=32)\end{array}$ & $\begin{array}{l}\text { Transdermal gel } \\
\qquad(n=6)\end{array}$ & & \\
\hline $\mathrm{T}(\mathrm{ng} / \mathrm{dl} ;$ median (IQR)) & $878.9(527.1-1161.0)$ & 549.75 (393.9-1033.2) & 807.7 (574.4-1405.5) & 0.11 & $321-1005$ \\
\hline FT (ng/dl; median (IQR)) & $20.1(13.8-30.2)$ & $13.5(8.1-25.5)$ & $20.7(15.7-38.2)$ & 0.11 & $6-25$ \\
\hline SHBG (nmol/l; mean (s.D.)) & $31.0(8.9)$ & $31.0(11.9)$ & $30.8(11.8)$ & 0.91 & $16-61$ \\
\hline LH (U/l; median (IQR)) & $6.8(0.1-17.5)$ & $3.5(0.44-28.4)$ & $20.5(0.4-49.1)$ & 0.50 & $1-9$ \\
\hline Hematocrit (\%; mean (S.D.)) & $48.5(2.2)$ & $49.0(2.8)$ & $46.1(2.4)$ & 0.078 & $39.8-52.2$ \\
\hline
\end{tabular}

IQR, interquartile range. To convert testosterone and FT to nanomoles per liter, multiply by 0.0347 .

${ }^{\text {aKruskall-Wallis test. }}$

${ }^{\mathrm{b}}$ Reference values based on males values at local laboratory.

we explored the associations between sexual desire scores and LH by ANOVA: this shows an independent association of LH on solitary and dyadic sexual desire after adjustment for age and weight $(P<0.001$; $P=0.024$ respectively).

Post hoc analysis showed that female-to-male transsexual persons with low LH levels have significantly higher levels of solitary sexual desire than participants with higher LH levels (Fig. 2). Participants with LH levels above P75 reported a significantly lower solitary sexual desire than those with LH levels below P25, but a similar level to those with LH levels within the P25-75 range. Post hoc analysis for dyadic sexual desire demonstrated only a significant difference between those with LH levels above P75 and P50-75 $(P=0.046)$ and below P25 and P50-75 $(P=0.011)$.

LH levels were also inversely associated with a higher frequency of experiencing excessive sexual desire (ANOVA; $P=0.007$ ). Post hoc analysis showed that participants with LH levels below P25 reported more frequent excessive sexual desire than participants with LH levels between P25 and 50 (LSD; $P=0.001$ ) or $\mathrm{LH}$ levels above P75 (LSD; $P=0.001$ ). LH levels were also inversely associated with a shorter time of being able to live without any sexual activities (ANOVA; $P=0.009$ ). Participants with LH levels below P25 reported having a higher need of sexual activities than those with LH levels between $\mathrm{P} 50$ and 75 (LSD; $P=0.004$ ) or LH levels above P75 (LSD; $P=0.005)$.

\section{Discussion}

Almost three quarters of our participants mentioned an increased sexual desire after SRS. This could be attributed to many changes associated with a sex reassignment procedure: improvement in general well being due to relief of gender dysphoria, testosterone therapy, or more satisfactory sexual relationships after SRS. Furthermore, the majority of the participants currently in a relationship reported a satisfactory sexual life. These findings are in line with previous studies that generally found an improvement in sexual functioning after SRS $(7,9,10,14)$.
To our knowledge, this is the first study investigating sexual desire in female-to-male transsexual persons using a validated questionnaire. We found a correlation between the desire to behave sexually by oneself (solitary sexual desire) and frequency of masturbation, but no relation between the desire to behave sexually with a partner (dyadic sexual desire) and frequency of sexual activities with a partner.

Female-to-male transsexual persons experience significantly higher frequency and intensity of sexual desire than male-to-female transsexual persons, a finding also reported in literature on sexual desire differences in biological men and women $(16,17)$. The higher level of androgens after sex reassignment in comparison with male-to-female transsexual persons might be one explanation for this finding. However, many biological, psychological as well as socio-cultural factors have been proposed to explain gender differences in sexual desire and motivation $(18,19)$.

With regard to the hormonal levels, we observed no statistical differences among the levels of total testosterone, calculated free testosterone, LH, and hematocrit according to the type of testosterone replacement therapy. However, borderline significance is observed

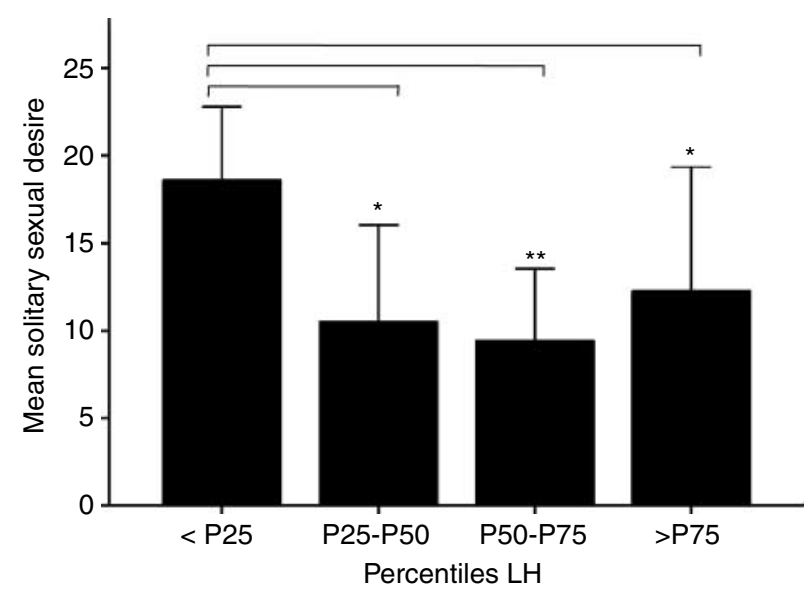

Figure 2 Solitary sexual desire inventory scores by different LH percentiles. Data is presented as mean \pm s.D.; ANOVA post hoc test (LSD). ${ }^{\star} P<0.01,{ }^{\star \star} P<0.001$. 
for (free) testosterone and hematocrit. The differences in sample size according to the type of testosterone therapy as well as the limited power of this study should be considered in this regard. Almost 9\% of our participants reached the cut off levels of hypogonadism. This finding can be explained as blood sampling was performed regardless of the moment of last administration of testosterone (testosterone undecanoate $1000 \mathrm{mg}$ median: 37 days, range: 1-97 days and parental testosterone esters $250 \mathrm{mg}$ median: 9 days, range: 1-30 days). However, the higher LH levels measured in these participants indicate the need to optimally adjust testosterone dosage. On the contrary, more than a quarter of our participants exceeded the upper limit of $1008 \mathrm{ng} / \mathrm{dl}$.

In this study, we could not establish a direct association between sexual desire and testosterone levels in female-to-male transsexual persons. However, we found an inverse association between solitary sexual desire and LH levels. Similarly, Van kesteren et al. (20) in their study on the effect of sex steroid treatment on bone health of transsexual persons observed that $\mathrm{LH}$ was a more adequate indicator than the levels of sex steroids themselves.

Inadequate testosterone therapy, as indicated by high LH levels were associated with lower levels of solitary sexual desire compared with participants with low LH levels, but not than those with $\mathrm{LH}$ levels within the normal range.

Participants with LH levels below P25 (all below the lower limit of our laboratory indicating an excess of testosterone replacement therapy) scored significantly higher on solitary sexual desire than those with LH levels within the normal range. Furthermore, participants with LH levels below P25 reported more often an excessive sexual desire and a having greater need for sexual activities than the others.

The reasons for not establishing a direct association between sexual desire and testosterone in femaleto-male transsexual persons could be multiple. First, the differences in sample size between the types of testosterone therapy in our participants could have hampered finding a relation, especially considering the relatively small sample size.

Secondly, the response to testosterone supplementation may differ individually $(21,22)$, further complicating finding an association. Thirdly, several studies have indicated that testosterone levels above a certain threshold value of testosterone do not have a significant impact on sexual functioning (23-26). Fourthly, serum androgen levels are only one aspect in the androgen cascade. Other factors, such as genetic variation of the transcriptional activity induced by the androgen receptor may modify testosterone action $(27,28)$.

Hormonal therapy in female-to-male transsexual persons aims at testosterone levels within the normal male physiological range (29). Adequate hormone therapy in female-to-male transsexual persons is needed to maintain for instance bone mass $(20,30)$. However, supraphysiological doses of testosterone increases the risk of adverse effects as erythrocytosis and liver dysfunction (31). Notably, the findings of our study reveal the importance of adequate testosterone therapy for a satisfactory sexual life since suppressed LH levels are associated with increased frequency of excess of sexual desire and higher LH levels with lower measures of sexual desire. Further investigation of these observations in larger prospective samples are needed to confirm our findings as well as investigating the clinical relevance of experiencing high or low levels of sexual desire, as could be indicated by the levels of distress.

Certain limitations of this study should be noted. First, the power of this study was limited, despite the fact that we were able to study a substantial number of female-to-male transsexual persons. Secondly, the crosssectional design implies that no causal relations could be drawn. Thirdly, we could only establish a difference in sexual desire between participants with LH below P25 and the others. We found no difference between participants with LH levels above P75 and LH levels between P25-50 and P50-75. However, the high variance in sexual desire scores in the group with $\mathrm{LH}$ levels above P75 as well as the limited power of this study should be considered. Also, selection bias of our study is another possible limitation. As in all follow-up studies, participants who agreed to this study may have a more favorable outcome than those who refused to participate. Nevertheless, the response rate is relatively high with $64 \%$ of those contacted willing to participate in a study that required a full day hospital visit. Finally, sexual functioning after SRS is partly dependent on the quality of and satisfaction with surgery that hampers generalization to all centers.

In conclusion, the data of this study indicate the majority of the participants reported an increase in sexual desire after cross-sex hormone treatment and SRS. No direct associations between levels of testosterone and measures of sexual desire were found. However, measures of sexual desire were inversely associated with LH levels.

\section{Declaration of interest}

The authors declare that there is no conflict of interest that could be perceived as prejudicing the impartiality of the research reported.

\section{Funding}

This research did not receive any specific grant from any funding agency in the public, commercial or not-for-profit sector.

\section{Acknowledgements}

The authors are indebted to Kaatje Toye for her help and assistance in the completion of this study. We also want to express gratitude to other members of our gender team: Piet Hoebeke, Steven Weyers, Gunter Heylens, Griet De Cuypere, and Stan Monstrey. 


\section{References}

1 Bancroft J. The endocrinology of sexual arousal. Journal of Endocrinology 2005186 411-427. (doi:10.1677/joe.1.06233)

2 Boloña ER, Uraga MV, Haddad RM, Tracz MJ, Sideras K, Kennedy CC, Caples SM, Erwin PJ \& Montori VM. Testosterone use in men with sexual dysfunction: a systematic review and metaanalysis of randomized placebo-controlled trials. Mayo Clinic Proceedings 200782 20-28. (doi:10.4065/82.1.20)

3 Isidori AM, Gianetta E \& Greco EA. Effects of testosterone on sexual function in men: results of a meta-analysis. Clinical Endocrinology 200563 381-394. (doi:10.1111/j.1365-2265.2005.02350.x)

4 Klein C \& Gorzalska BB. Sexual functioning in transsexuals following hormone therapy and genital surgery: a review. Journal of Sexual Medicine 20096 2922-2939. (doi:10.1111/j. 1743-6109.2009.01370.x)

5 Elaut E, De Cuypere G, De Sutter P, Gijs L, Van Trotsenburg M, Heylens G, Kaufman JM, Rubens R \& T'Sjoen G. Hypoactive sexual desire in transsexual women: prevalence and association with testosterone levels. European Journal of Endocrinology 2008158 393-399. (doi:10.1530/EJE-07-0511)

6 Kronawitter D, Gooren LJ, Zollver H, Oppelt PG, Beckmann MW, Dittrich R \& Mueller A. Effects of transdermal testosterone or oral dydrogesterone on hypoactive sexual desire disorder in transsexual women: results of a pilot study. European Journal of Endocrinology 2009161 363-368. (doi:10.1530/EJE-09-0265)

7 De Cuypere G, T'Sjoen G, Beerten R, Selvaggi G, De Sutter P, Hoebeke P, Monstrey S, Vansteenwegen A \& Rubens R. Sexual and physical health after sex reassignment surgery. Archives of Sexual Behavior 200534 679-690. (doi:10.1007/s10508-0057926-5)

8 Lief H \& Hubschman L. Orgasm in the postoperative transsexual. Archives of Sexual Behavior 199322 145-155. (doi:10.1007/ BF01542363)

9 Smith YLS, Van Goozen SHM \& Cohen-Kettenis PT. Adolescents with gender identity disorder who were accepted or rejected for sex reassignment surgery: a prospective follow up study. Journal of the American Academy of Child and Adolescent Psychiatry 200140 472-481. (doi:10.1097/00004583-200104000-00017)

10 Cohen-Kettenis PT \& Van Goozen SHM. Sex reassignment of adolescent transsexuals: a follow-up study. Journal of the American Academy of Child and Adolescent Psychiatry 199736 263-271. (doi:10.1097/00004583-199702000-00017)

11 Spector IP, Carey MP \& Steinberg L. The Sexual Desire Inventory: development, factor structure, and evidence of reliability. Journal of Sex and Marital Therapy 199622 175-190. (doi:10.1080/ $00926239608414655)$

12 Monstrey S, Hoebeke P, Dont M, Selvaggi G, Hamdi M, Van Landuyt K \& Blondeel P. Radial forearm phalloplasty: a review of 81 cases. European Journal of Plastic Surgery 200528 206-212. (doi:10.1007/s00238-005-0743-1)

13 Monstrey S, Hoebeke P, Selvaggi G, Ceulemans P, Van Landuyt K, Blondeel P, Hamdi M, Roche N, Weyers S \& De Cuypere G. Penile reconstruction: is the radial forearm flap really the standard technique? Plastic and Reconstructive Surgery 2009124 510-518. (doi:10.1097/PRS.0b013e3181aeeb06)

14 Wierckx K, Van Caenegem E, Elaut E, Vandepeer F, Dedecker D, Toye K, Weyers S, Hoebeke P, Monstrey S, De Cuypere G \& T'Sjoen G. Quality of life and sexual health after sex reassignment surgery in transsexual men. Journal of Sexual Medicine, 2011. In press.

15 Vermeulen A, Verdonck L \& Kaufman JM. A critical evaluation of simple methods for the estimation of free testosterone in serum. Journal of Clinical Endocrinology and Metabolism $1999 \quad \mathbf{8 4}$ 3666-3672. (doi:10.1210/jc.84.10.3666)

16 Laumann EO, Paik A \& Rosen RC. Sexual dysfunction in the United States. Prevalence and predictors. Journal of the American Medical Association 1999281 537-544. (doi:10.1001/jama.281. 6.537)

17 Laumann EO, Nicolosi A, Glasser DB, Paik A, Gingell C, Moreira E \& Wang T. Sexual problems among women and men aged 40-80 y: prevalence and correlates identified in the Global Study of Sexual Attitudes and Behaviors. International Journal of Impotence Research 200517 39-57. (doi:10.1038/sj.ijir.3901250)

18 Marks M \& Fraley R. Confirmation bias and the sexual double standard. Sex Roles 200654 19-26. (doi:10.1007/s11199-0068866-9)

19 Baumeister R, Cathanese K \& Vohs K. Is there a gender difference in strength of sex drive? Theoretical views, conceptual distinctions, and a review of relevant evidence Personality and Social Psychology Review $2001 \quad 5 \quad 242-273$. (doi:10.1207/ S15327957PSPR0503 5)

20 Van Kesteren P, Lips P, Gooren LJ, Asscheman H \& Megens J. Long-term follow-up of bone mineral density and bone metabolism in transsexuals treated with cross-sex hormones. Clinical Endocrinology 199848 347-354. (doi:10.1046/j.1365-2265. 1998.00396.x)

21 Bhasin S, Woodhouse L, Casaburi R, Singh AB, Bhasin D, Berman N, Chen X, Yarasheski KE, Magliano L, Dzekov C, Dzekov J, Bross R, Phillips J, Sinha-Hikim I, Shen R \& Storer TW. Testosterone dose-response relationships in healthy young men. American Journal of Physiology. Endocrinology and Metabolism 2001 281 1172-1181.

22 Buena F, Swerdloff RS, Steiner BS, Lutchmansingh P, Peterson MA, Pandian MR, Galmarini M \& Bhasin S. Sexual function does not change when serum testosterone levels are pharmacologically varied within the normal male range. Fertility and Sterility 1993 59 1118-1123.

23 Gooren LJ. Androgen levels and sex functions in testosteronetreated hypogonadal men. Archives of Sexual Behavior 198716 463-473. (doi:10.1007/BF01541711)

24 Kelleher S, Conway AJ \& Handelsman DJ. Blood testosterone threshold for androgen deficiency symptoms. Journal of Clinical Endocrinology and Metabolism 200489 3813-3817. (doi:10. 1210/jc.2004-0143)

25 Bagatell CJ, Heiman JR, Rivier JE \& Bremner WJ. Effects of endogenous testosterone and estradiol on sexual behavior in normal young men. Journal of Clinical Endocrinology and Metabolism 199478 711-716. (doi:10.1210/jc.78.3.711)

26 Zitzmann M, Faber S \& Nieschlag E. Association of specific symptoms and metabolic risks with serum testosterone in older men. Journal of Clinical Endocrinology and Metabolism 200691 4335-4343. (doi:10.1210/jc.2006-0401)

27 Crabbe P, Bogaert V, De Bacquer D, Goemaere S, Zmierczak H \& Kaufman JM. Part of the inter individual variation in serum testosterone levels in healthy men reflects differences in androgen sensitivity and feedback setpoint: contribution of the androgen receptor polyglutamine tract polymorphism. Journal of Clinical Endocrinology and Metabolism 20079 3604-3610.

28 Zitzmann M, Gromoll J \& Nieschlag E. The androgen receptor CAG repeat polymorphism. Andrologia 200537 216. (doi:10.1111/j. 1439-0272.2005.00692.x)

29 Hembree WC, Cohen-Kettenis P, Delemarre-van de Waal H, Gooren LJ, Meyer WJ, Spack N, Tangpricha V \& Montori V. Endocrine treatment of transsexual persons: an Endocrine Society Clinical Practice Guideline. Journal of Clinical Endocrinology and Metabolism 200994 3132-3154. (doi:10.1210/jc.2009-0345)

30 Turner A, Chen TC, Barber TW, Malaba AO, Holick MF \& Tangpricha V. Testosterone increases bone mineral density in female-to-male transsexuals: a case series of 15 subjects. Clinical Endocrinology 200461 560-566. (doi:10.1111/j.1365-2265. 2004.02125.x)

31 Bhasin S, Cunningham GR, Hayes FJ, Matsumoto AM, Snyder PJ, Swerdloff RS \& Montori VM. Testosterone therapy in adult men with androgen deficiency syndromes: an Endocrine Society Practical Guideline. Journal of Clinical Endocrinology and Metabolism 200691 1995-2010. (doi:10.1210/jc.2005-2847)

Received 18 May 2011

Accepted 19 May 2011 